\title{
Determination of sialic acid in saliva by means of surface-enhanced Raman spectroscopy as a marker in adnexal mass patients: ovarian cancer vs benign cases
}

José de Jesús Zermeño-Nava ${ }^{1}$, Marco Ulises Martínez-Martínez ${ }^{1,4}$, Ana Laura Rámirez-de-Ávila1, Aida Catalina Hernández-Arteaga², Ma. Guadalupe García-Valdivieso ${ }^{2^{*}}$ (D), Alondra Hernández-Cedillo², Miguel José-Yacamán ${ }^{2,3}$ and Hugo Ricardo Navarro-Contreras ${ }^{2^{*}}$

\begin{abstract}
Background: To demonstrate the use of surface-enhanced Raman spectroscopy (SERS) to determine sialic acid (SA) levels in saliva using silver nanoparticles as substrates, in adnexal mass patients scheduled for surgical intervention to remove invasive masses, with the aim to compare SA levels in benign tumor vs ovarian cancer patients.

Methods: Quantification of SA levels was accomplished by measuring their SERS and calibrating with analytical reagent SA. The mean SA concentration in saliva from 37 benign adnexal mass resulted smaller $(5.1 \mathrm{mg} / \mathrm{dL})$ than the mean concentration in 15 Ovarium cancer patients $(23 \mathrm{mg} / \mathrm{dL})$. The cancer condition was determined by biopsy of the removed adnexal mass. The CA-125 biomarker was also measured. The predictive potential of both biomarkers is discussed, together with the malignity risk index (MRI).
\end{abstract}

Results: Our results showed a sensitivity/specificity of 80\%/100\% with a cutoff to distinguish between benign/cancer cases of SA $15.5 \mathrm{mg} / \mathrm{dL}$, as established from a ROC analysis. Our results suggest that SA may be a more useful biomarker than CA-125 to detect ovarian cancer.

Conclusions: Our results suggest that the SA levels measured from saliva may be as good predictors as the MRI index for the presence of ovarian cancer in sensitivity/negative predictive value and outperforms it in specificity/positive predictive value.

Keywords: Sialic Acid, Surface-enhanced Raman, Ag Nanoparticles, Ovarian Cancer

\section{Background}

Ovarian cancer is the seventh most common cancer in women worldwide [1, 2]. In 2012239,000 ovarian cancer cases were reported; which amounts to $4 \%$ of all new cases of cancer in women. Ovarian cancer produced approximately 152,000 deaths in 2012 . It is the eighth most common cause of cancer death in women across the world $[1,2]$.

\footnotetext{
* Correspondence: guadalupe.valdivieso@uaslp.mx; hnavarro@uaslp.mx ${ }^{2}$ Coordinación para la Innovación y Aplicación de la Ciencia y la Tecnología (CIACYT), Universidad Autónoma de San Luís Potosí, Álvaro Obregón 64, 78000 San Luis Potosí, SLP, Mexico

Full list of author information is available at the end of the article
}

Ovarian cancer (OC), in general do not produce symptoms at early stages. Additionally, there is no early detection method applicable to the general women population, so the disease is generally advanced when it is diagnosed. Ovarian epithelial cancer is the histological type of major incidence, representing almost $90 \%$ of reported cases. In more than $70 \%$ of all cases, it is usually detected in the advanced clinical stages III and IV. The 5 year survival rate ranges from approximately 30 to $50 \%$ depending on the type of ovarian cancer, being the invasive epithelial ovarian cancer the most common, as well as that of the worst prognosis, in phase III-IV patients, which have a $5-20 \%$ five year survival rate $[3,4]$.

(c) The Author(s). 2018 Open Access This article is distributed under the terms of the Creative Commons Attribution 4.0 International License (http://creativecommons.org/licenses/by/4.0/), which permits unrestricted use, distribution, and 
This high mortality rate prompts the need to have effective, highly sensitive diagnostics methods to examine women who may have evidence of ovarian cancer, or symptoms who may lead the medical specialist to consider further clinical examination of the involved patient, to discard the existence of ovarian cancer.

For the medical diagnosis, there is a limited set of analysis that may help to detect and diagnose ovarian cancer. Transvaginal ultrasound is the most useful imaging method for routine screening of ovarian cancer, given that this cancer is usually only palpable in advanced stages. The most important biomarker that has proven to be a useful adjunct for ovarian cancer is the protein CA-125, which has been found is produced by more than $90 \%$ of advanced epithelial ovarian cancers [3, 4]. Normal values range from 0 to $35(\mathrm{U} / \mathrm{mL})$. As a result, the CA-125 protein has become the most screened serum marker in laboratory tests for ovarian cancer. However, although CA-125 significantly rises 4 months previous to any clinical manifestation, in almost $50 \%$ of the early screenings of positive cases, it results in normal concentration levels [5]. This fact reduces the usefulness of CA-125 as a predictive test for this malignancy. Additionally, for young patients (premenopausal women) there is not any clearly defined cutoff point for this biomarker which can be associated with any malignity risk with certainty.

Considering these limitations, as well as the fact that ovarian cancer is of relative low prevalence, screening of the general sensitive female population for ovarian is not recommended [5], although less than $30 \%$ of patients with ovarian cancer are diagnosed at an early curable stage [6]. Instead, an annual gynecological exam with pelvic examination is recommended for preventive health care.

One major problem is that screening methods tend to find a large number of false positive cases. The combined use of transvaginal ultrasound and CA-125 tend to result in a higher sensitivity for ovarian cancer detection, but at an increased rate of false positive results $[5,6]$. Unfortunately, malignancy can only be assessed with surgery. These could lead to unnecessary surgery in false cases, with the additional full inconveniency of the probability of complications due to surgery, as well as anxiety caused by false alarming results.

Another biomarker for ovarian cancer screening that has been studied and proposed is the Human Epididymis Protein 4 (HE4) [7]. HE4 is a tumor marker of ovarian cancer, with $80 \%$ sensitivity at a cutoff of $150 \mathrm{pmol} / \mathrm{L}$ [7]. However, it is not routinely used by the medical professionals in standard medical tests to screen for the presence of this disease.

Saliva has been proposed as an alternative medium to plasma in monitoring biomarkers that may be useful in diagnosis of several human diseases, as well as an element to monitor the etiology of some pathologies or the effects of drugs doses. The simplicity and non-invasiveness of its recollection, the existing positive correlation among multiple substances that are present in serum and saliva, are some of the advantages that this fluid offers as a diagnostic instrument for some human diseases $[8,9]$.

An important biomarker in saliva is sialic acid (SA), which is a systematic biomarker of systemic inflammation [10], it is a component bound to salivary glycolipids, and to glycoproteins, of which IgA (immunoglobuline A) may be one of the most important, as well as from some other immunologic proteins. SA occupies the interface between a host and pathogenic microorganisms.

Sialic acids are a family of nine-carbon acidic monosaccharides that occur at the end of oligosaccharide chains of mucins, glycoproteins, and glycolipids attached to the cell membranes of all vertebrate organisms [10]; SA emerging from these membranes is continuously shed into the surrounding human fluids. $\mathrm{N}$-acetylneuraminic acid (Neu5AC) is the predominant form of SA and almost the only form found in human body fluids and tissues, among them saliva [10]. It has the chemical formula $\mathrm{C}_{11} \mathrm{H}_{19} \mathrm{NO}_{9}$. The molecule is composed of a pyranose ring consisting of five carbon atoms and one oxygen atom, to which one $\mathrm{N}$-acetyl, one carboxyl group, and one glycerol ion are attached.

SA is normally detected and calibrated in its concentration in human fluids, by decanting it by an elaborated chemical process to isolate it from other proteins or non-related lipids which are constitutive of these fluids. Followed by comparison of its absorbance at some given wavelengths with standard calibration curves obtained from chemically pure reagents $[11,12]$. Recently, we have shown that small amounts of SA in aqueous solution may be easily detected by the alternative method of surface enhanced Raman spectroscopy (SERS) [13], produced by citrate-reduced silver nanoparticles (cit-Ag-NP), with a simplified processing of the saliva sample. We have demonstrated the sensitivity of this method to distinguish between breast cancer patients and healthy control subjects [14]. This is possible as SA is well known to be over expressed in human fluids when there is an ongoing cancer development process. Determination of SA by SERS from cit-Ag-NP is a technological approach that requires fewer reagents, and simplified saliva sample processing. The latter constitutes an advantage that could be useful in clinical diagnosis since it is highly sensitive, fast, and low cost, moreover, the equipment may be portable and results can be obtained in real time.

SERS is a Raman spectroscopic technique that has shown enhancements of the inelastic scattering of the outgoing radiation ranging from $10^{6}$ to $10^{12}$ in amplification factors from molecules that have been adsorbed in metals [15]. Therefore, SERS has great potential as a molecularly specific analytic probe for highly sensitive 
detection of weak Raman signals of proteins or other biological analytes with low Raman scattering cross-section or at very low concentrations. The threshold of concentrations detectable may be as low as a single-molecule attached to a Ag-NP [15]. Furthermore, colloidal suspensions of metallic nanoparticles (NP), silver, gold or copper are the most common SERS substrates because they are of easy preparation and have long shelf-lifetime, and high Raman signal enhancement factors [16].

A primary aim of this study is to apply SERS to measure levels of SA in human saliva using colloidal suspension of cit-Ag-NP, and in particular to determine SA levels in saliva of patients to whom adnexal masses in ovary have been observed by ultrasound sonography, and who have been scheduled for surgical removal of the detected tumor.

\section{Hypothesis}

The hypothesis is that there may exist a significant difference in SA levels which have been expressed in saliva, as in many other human fluids, between benign tumor cases and ovarian cancer affected patients.

Our main objective was to establish the usefulness of monitoring the SA levels by SERS from cit-Ag-NP, in suspected cases of ovarian cancer, thus adding the SA level tests as another adjunct probe to the diagnosis of ovarian cancer, together with CA-125 and ultrasound imaging. SERS has the advantages of its simplicity and may be extremely useful when it is necessary to monitor the progression of the disease as well as monitor the effectiveness of applied treatments. Our results corroborate that salivary SA concentrations are found to be significantly higher in ovarian cancer patients compared to that of benign adnexal mass patients.

\section{Methods}

\section{The study population}

Inclusion criteria: Patients attending the service of the Gynecology and Obstetrics at Central Hospital Dr. "Ignacio Morones Prieto" at San Luis Potosí, S.L.P. México, (HCDSLP) during the periods July-December 2017 , which were diagnosed by ultrasound to have adnexal tumor masses and that were scheduled for surgical intervention to remove them, with no previous clinical treatment.

Exclusion criteria: any previous cancer diagnosis, second primary cancer, pregnancy, any systemic or circulatory diseases, or periodontal disease.

The study was approved by the HCDSLP Ethics Committee. Written informed consent was obtained from all participants.

\section{Cancer determination}

Histological type determined by the Pathology laboratory at the Central Hospital "Dr. Ignacio Morones Prieto" from tumor biopsy.

\section{Data collection}

Adnexal tumor patients completed a health questionnaire that included information about systemic health and oral diseases. Blind (regarding the existence of ovarian cancer) determination of the SA concentration (SAC) in saliva and CA-125 in serum were made.

\section{Saliva collection and processing}

Before the saliva collection, each participant was required to perform a two-step oral cleansing. The first step consists of a vigorous teeth brushing and the second one involves two subsequent oral rinses with commercial alcohol-free mouthwash. The saliva samples were centrifuged at $6000 \mathrm{rpm}$ (equivalent to $3580 \mathrm{G}$ ) for $15 \mathrm{~min}$, and the resulting supernatants were used to determine the concentration of SA.

\section{SERS calibration of SA}

The Raman measurements were performed on a Horiba Jobin Yvon XploRA ONE Raman spectrometer coupled to an Olympus BX41 optical microscope, using an excitation green laser $532 \mathrm{~nm}$, with an average power of $20 \mathrm{~mW}$ at the sample. The laser beam was focused on the surface of the liquid sample with a $10 \mathrm{X}$ objective. The diameter of the laser spot was approximately 8$10 \mu \mathrm{m}$. The SERS spectra were collected from the 400 to $1800 \mathrm{~cm}^{-1}$ spectral range. The intensity of three Raman shift lines, 1002, 1237 and $1391 \mathrm{~cm}^{-1}$, after fluorescence subtraction, are used to compare with a calibration curve for the SERS obtained from SA Analytical Reagent grade. Next, to record the SERS spectra, $50 \mu \mathrm{l}$ of a $2.5 \times 10^{-3} \mathrm{M}$ citrate-reduced Ag-NP were placed in an aluminum container, $100 \mu \mathrm{l}$ in capacity, mixed with $25 \mu \mathrm{l}$ of the centrifuged saliva sample. An equal volume of a reference SA solution was using in the calibration process previous to any measurement session. Further details on the calibration process are provided in [14].

\section{Data statistical analysis}

Continual variables are reported using the median \pm interquartile range (IQR) because of the non-normal distribution of some of them, as discussed below. Categorical variables are reported as $n$ and percentage. Non-parametric tests were performed to analyze the continual variables (Mann Whitney U test). The categorical variables were analyzed with the Fisher exact tests. The statistical analysis was carried out with the statistical program R 3.4.3 (R Core Team 2017), and RStudio Version 1.1.423. Receiver operating characteristics (ROC) curves were plotted to calculate 
the area under the curve and to establish the best point for sensitivity and specificity.

\section{Results and discussion}

Fifty-two patients that were diagnosed with adnexal masses scheduled for surgical intervention, age ranging from 25 to 78 years old (average: 41.6 years \pm 17.3 ) participated in this study. The characteristics of the patients participating in the project are shown in Table 1. One may see that the histological analysis resulted in 37 patients with benign adnexal masses (mean age and S.D. 36.8 years \pm 15.3 ). In 15 patients (mean age and S.D. 51.2 years \pm 18.7 ), these masses were ovarian cancer.

A Shapiro test for normality in the distribution of the measured variables was performed. In the test, a significance level below $p<0.05$ indicates that the variables are not normally distributed. Only body mass index and Menarche were normally distributed, the SA and CA-125 data require a non-parametric statistical analysis. Given the fact that most variables are non-parametric, we proceeded to evaluate the median plus/minus the first and third quartiles $(Q 1, Q 3)$, which are summarized in Table 1 for the relevant parameters SAC and CA-125.

Figure 1 shows the sialic acid concentration (SAC) of each group of patients. Indicated in black, are the SAC of benign adnexal mass (BAMP) affected patients, and in red those in which histology has diagnosed ovarian cancer (OCP). The inset in Fig. 1 shows the boxplot of sialic acid concentration in both groups.

The difference between concentration means of benign adnexal mass patients and positive diagnosis ovarian cancer cases was assessed by a Mann Whitney $U$ test, which has been also summarized in Table 1 . A $P \leq 0.05$ value was considered to be statistically significant. The application of the Mann Whitney $U$ test supports the hypothesis that the expression of SA that is present in the saliva of benign and ovarian cancer patients is statistically different.

Table 1 shows that there are no expected statistical differences in the BMI, menarche and gestations variables between the two groups, BAMP and OCP. However, the same analysis indicates that there are significant statistical differences in both CA125 and SAC, between the two groups of patients.

Figure 2 shows the result of a Receiver Operating Characteristic or ROC curve analysis for the sialic acid concentrations between the two group of patients. As always the axes are chosen vertical for Sensitivity (true positive rate), and horizontal 1-Specificity (false positive rate). This analysis performed with the R-Studio program results in that the best threshold to differentiate between BAMP and OCP corresponds to $\mathrm{SAC}>15.5 \mathrm{mg} / \mathrm{dL}$, rounded to one decimal digit. The area under the ROC curve is $94.05 \%$ for the SAC cutoff proposed. This SAC threshold value corresponds to a sensitivity of $80 \%$, and specificity of $100 \%$. The figure has also included the ROC analysis for the CA-125. This analysis results in a smaller area under the curve of $82.52 \%$, as compared to the SAC. The resultant suggested cutoff from the ROC analysis for the CA-125 is $216.5 \mathrm{U} / \mathrm{mL}$. This is a very high concentration value, corresponding to a high specificity value of $94.5 \%$, but to a poor sensitivity because it fails to identify at least 8 out of the 15 positive cases (more than $50 \%$ of the cases).

Figure 3 shows a CA-125 vs SA plot of the benign adnexal mass and ovarian patients, (black and red points, respectively). The Spearman correlation coefficient among CA-125 and SAC is 0.56, indicative of a modest correlation among the expression of these two biomarkers. The blue colored lines depict the standard cutoff concentrations for the CA-125 (horizontal dashed line, $35 \mathrm{U} / \mathrm{mL}$ ) and the proposed SAC cutoff (vertical dashed line). The

Table 1 Patients that have been diagnosed with adnexal masses characteristics that were included in the study

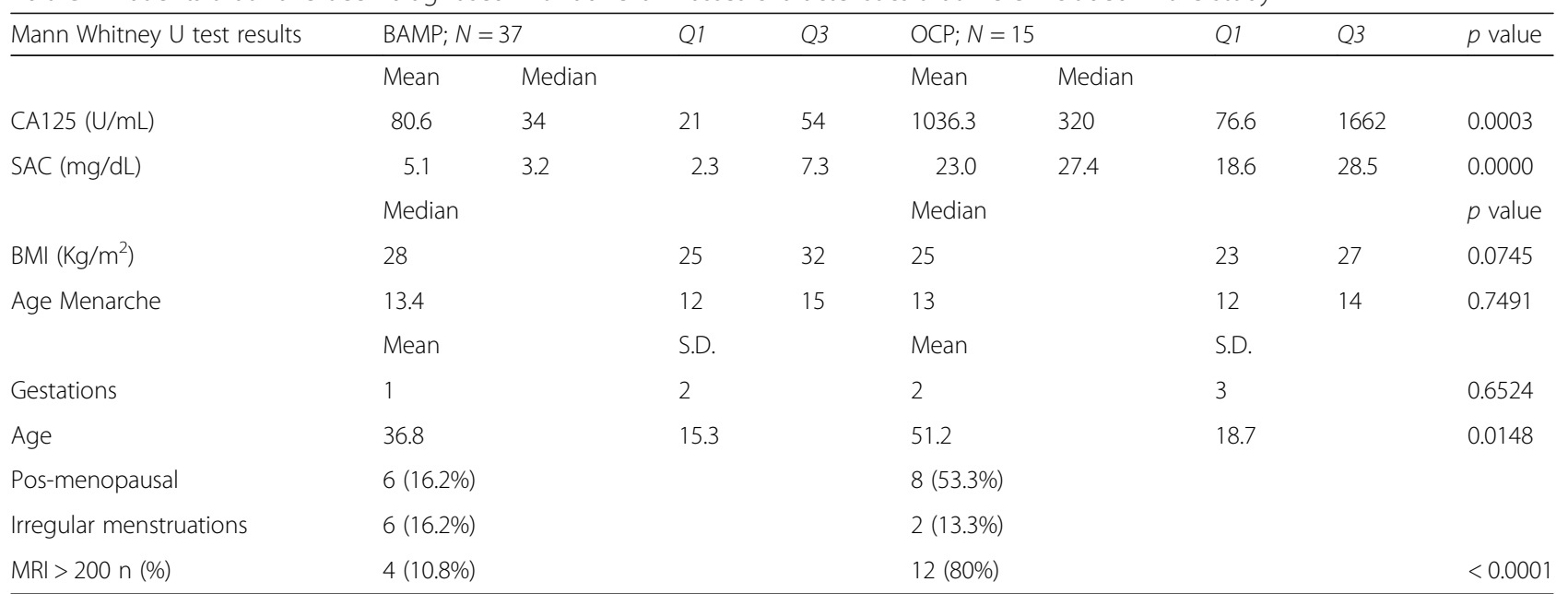

The $p$ stands for the significance obtained for BAMP benign adnexal mass and OCP ovarian cancer affected patients, under the Mann Whitney $\mathrm{U}$ tests 


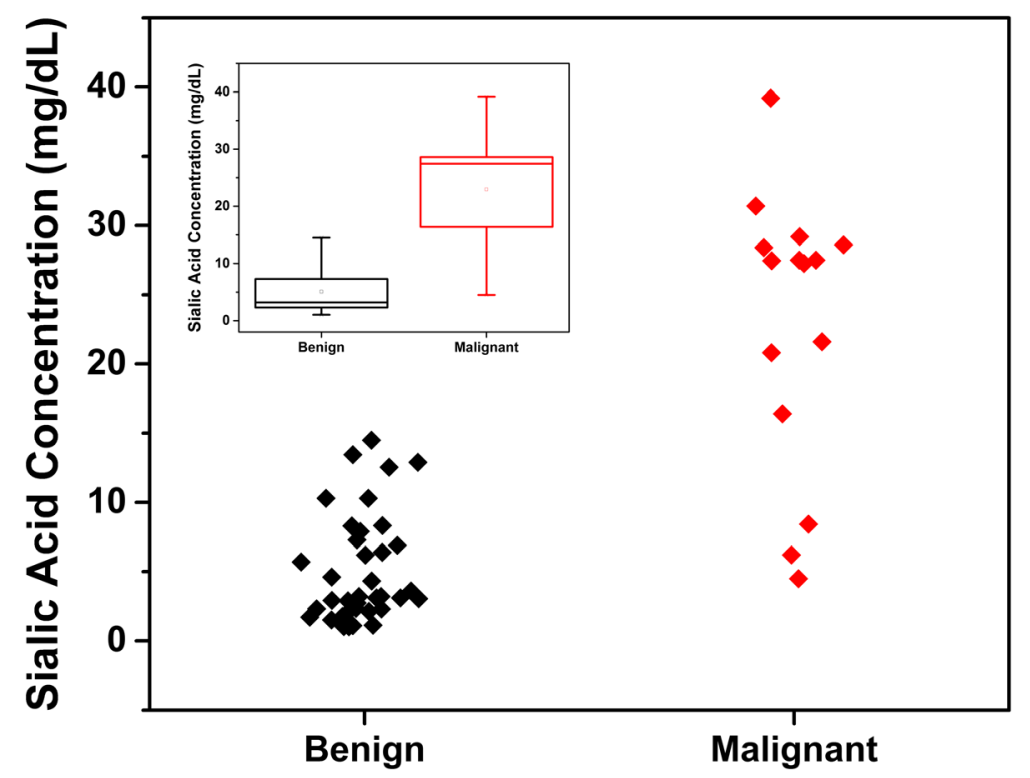

Fig. 1 Cloud plot of sialic acid concentration of each group of patients. Red: ovarian cancer; black: benign adnexal mass. Insert: Box plot of sialic acid concentration, again red: ovarian cancer; black: benign adnexal mass

CA-125 concentrations are plotted on a logarithmic scale, in order to have a better visualization of the spreading in resultant patient concentrations that were determined for this biomarker. From the plot, it is evident that SA under the cutoff provided by the ROC analysis has a better performance than CA-125 to distinguish between benign and ovarian cases. But with respect to detecting true positives, CA-125 in this exercise missed only two positive cases, at the $35 \mathrm{U} / \mathrm{ml}$ cutoff, vs 3 for SA, but at the expense of resulting in too many false positives, 15 of them in this study (almost $40 \%$ of benign cases), against none for the $\mathrm{SA}$, under the cutoff of $15.5 \mathrm{mg} / \mathrm{dL}$.

Figure 4, presents a comparison between the sensitivities, specificities positive predictive values (PPV) and negative predictive values (NPV) for each test. The Figure includes the cutoff values and confidence intervals for

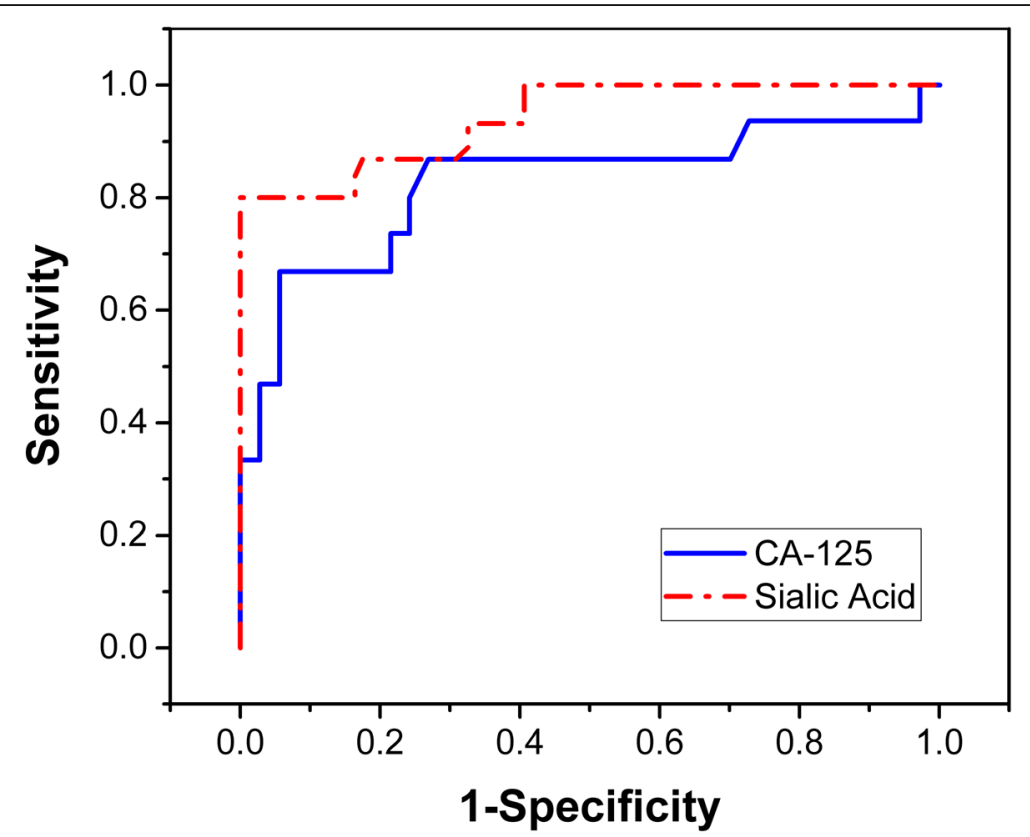

Fig. 2 ROC curve analysis for SA (and CA-125), to optimize the threshold of acid sialic concentrations to distinguish between benign adnexal mass patients, and ovarian cancer patients 


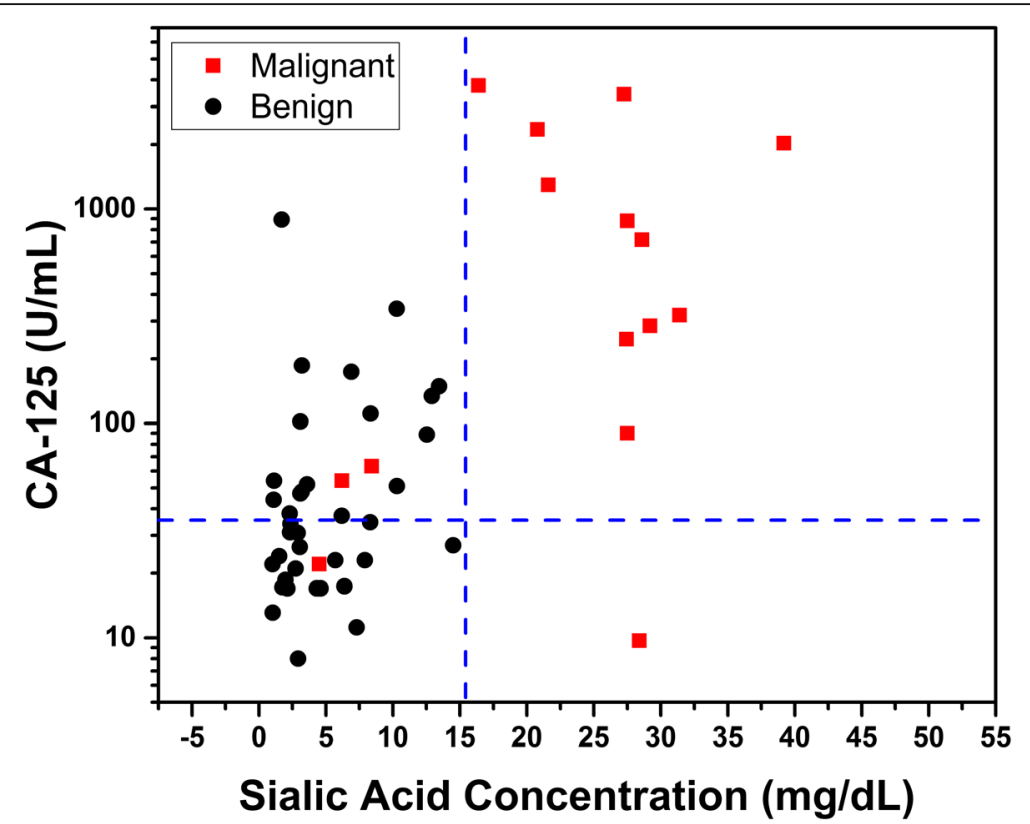

Fig. 3 SA and CA-125 plot, to illustrate how the cutoff values of SA and CA-125 concentrations distinguish between benign adnexal mass patients (black), and ovarian cancer patients (red)

each biomarker, as well as for the calculated MRI. The MRI was calculated following Jacobs et al. [17] $M R I=U \times M \times C A-125$. In this formula $M$ is the menopausal status score, and $U$ the evaluation of the ultrasound score [17]. The Figure shows how the
SAC under the cutoff ROC value of $15.5 \mathrm{mg} / \mathrm{dL}$ performs as well as the calculated MRI in sensitivity and negative predictive values and may be even superior in specificity and positive predictive values. The Figure includes the performance of CA-125 under the cutoff values

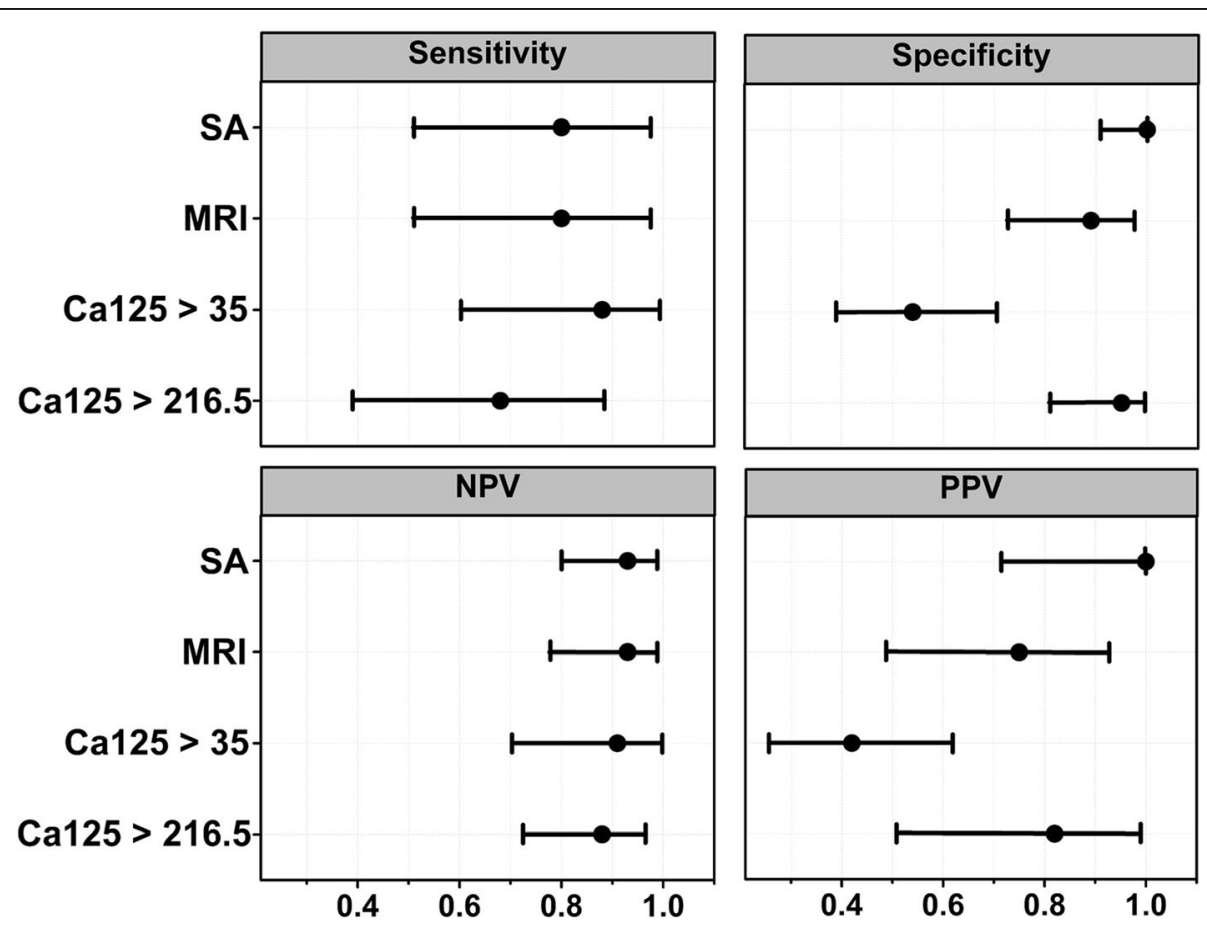

Fig. 4 Sensitivity, specificity, positive predictive value (PPV) and negative predictive value (NPV) for each test. The Figure includes cutoff values and confidence intervals for each biomarker, as well as for the calculated MRI. Abbreviations: SA, sialic acid; MRI, Malignancy risk, index, CA125 > 35, CA-125 larger than $35 \mathrm{U} / \mathrm{mL}$; CA125 > 216.5, CA-125 larger than $216.5 \mathrm{U} / \mathrm{mL}$ 
of 35 and the best result of the ROC analysis of our data of $216 \mathrm{U} / \mathrm{mL}$.

\section{Discussion}

The results indicate that with a cutoff or threshold value of $15.5 \mathrm{mg} / \mathrm{dL}$ of the SA concentration measured in saliva we have a $100 \%$ specificity ( $80 \%$ sensitivity). These results suggest that we could distinguish before surgery if an adnexal masses patient, as revealed through sonography, is going to have ovarian cancer with high probability, if the patient presents higher SA concentration levels than this cutoff value. Of course this analysis requires that patients are discarded of suffering any systemic or circulatory diseases, advanced periodontitis or from any other primary diagnosed cancer, to circumvent the intrinsic limitation that $\mathrm{SA}$ is a nonspecific marker of inflammation.

It is clear that although SA is not a specific marker for ovarian cancer, it should be considered in the clinical scenario of the adnexal mass diagnosed patient, and not in the population in general. In spite of the non-specificity for cancer, the values obtained in the tests suggest that they are sensitive and specific enough to differentiate an inflammatory process against a cancer process, when combined with the clinical evaluation by the medical professional.

It is important to note that in our study, the mean age of the benign adnexal mass group is 36.8 years old which is smaller than the mean age of the ovarian cancer group of age 51.2. It has been reported that the SA levels increases only by $4-5$ and $7 \%$ in the age groups $50-59$ and 60-69, compared to the 20-49 age group, in which the SA levels remain practically the same [18]. Hence, as the mean ages of the two groups fall below $<50$ years, any age effect is not expected to affect in any significant way the SAC data of the patients in this study.

The search for malignant tumor markers has been a permanent concern for the professionals of gynecological oncology [18]. In particular for Ovarium cancer Schwartz et al. in 1987, calibrating from absorbance curves, they found that lipid-associated sialic acid (LSA) levels in the serum of patients without any clinical evidence of disease were statistically different, from those patients with evidence of the disease [19]. The medians of LSA that they reported are of 18.4 and $27.2 \mathrm{mg} / \mathrm{dL}$, for those two groups of patients, respectively. Additionally, the Schwartz group [19] found that the simultaneous use of the LSA and CA-125 concentrations enhances significantly the sensitivity for Ovarium cancer detection, from $76 \%$ for CA- 125 alone to $84 \%$ when using information of the two markers combined, with cut off values of LSA $>24 \mathrm{mg} / \mathrm{dL}$ and CA-125>60 U/mL. A similar improvement is also reported for the specificity of both tests combined. Petru et al. [20], studied also the use of CA-125 and LSA extracted from serum, to test for the increase in sensitivity for diagnosing Ovarium cancer. However, in this study with the determination of both markers no significantly improved sensitivity was reported, in contrast to the use of CA-125 alone. But Petru et al. [20] reports very high sensitivities values for CA-125 (90.2\%) for diagnosing Ovarium cancer. These high values have not been replicated by other studies [21-23]. For instance, Li et al. [21], found that the combined determination of SA with hydroxyproline provides a better diagnosis value than either CA-125 or HE4. S. Ghosh [22] discusses in a recent work, how SA in serum may also be used as a clinical predictor for Ovarium cancer. Finally, we add that Thakkar et al. [23] found that protein-bound sialic acid (PBSA) provides the highest overall ability to discriminate between ovarian cancer patients and healthy subjects, in comparison with other glycoproteins in serum [23].

Our present work adds to those previous reports the value of SA as a predictor of possible Ovarium cancer presence in adnexial mass diagnosed patients when it is detected by SERS in saliva in larger concentrations than the cutoff value obtained in our ROC analysis of $15.5 \mathrm{mg} / \mathrm{dL}$. An important problem that our results for SAC in saliva may help to alleviate is the large number of unnecessary surgeries performed during the approach to treat the adnexal lesion. Our study is not a screening test since it was performed in a population with adnexal pathology, however our test of the over expression of SAC in saliva shows a very high specificity. We state the expectation that his may help to significantly decrease the number of unnecesary invasive surgical procedures in the population of these patients.

The present study was by necessity limited to the small group of adnexal mass patients expecting for treatment at HCDSLP during a six month period, and that were scheduled for surgical intervention to remove them. The very promising results for the SERS test determination of SAC from saliva, in specificity and sensitivity for ovarian cancer, determined in this work, suggest that it is necessary to continue the study to include a larger group of patients to corroborate our present results.

A necessary commentary in regards to SERS as a characterization technique of chemical biomarkers is that still has not been readily adopted by the clinical institutions. The only limitation that we perceive is that it is sensitive to the selection of prepared nanoparticles (NP), i.e. metal and full surface coverage of functionalization molecule types required to keep them in suspension, which require a careful study to find the most appropriated to the biomarker in consideration. But once these are established, the NP's are easily reproducible, with repetitive SERS properties. 


\section{Conclusions}

Our measurements of SA by SERS produced by citratereduced silver nanoparticles of ovarian cancer affected patients indicate that these levels are increased with respect to the median SA level in adnexal mass subjects, corroborating previous results in the literature, where SA has been measured by conventional absorbance methods, which require extensive chemical processing of the samples. The threshold to differentiate between benign adnexal mass and Ovarium cancer patients is found to be $\mathrm{SAC}>15.5 \mathrm{mg} / \mathrm{dL}$, from a ROC curve analysis. This SA level threshold or cutoff value corresponds to a sensitivity of $80 \%$, and specificity of $100 \%$. Our results suggest that the SA levels that are measured from saliva samples may be as good predictors as the MRI index for the presence of ovarian cancer in sensitivity and negative predictive value and may outperform it in specificity and positive predictive value.

The SERS method requires no chemical elaboration of the saliva samples, and could be useful in clinical diagnosis since it is highly sensitive, fast, inexpensive, the equipment may be portable and results can be obtained in real time. Thus, the SERS method to determine the SA level in saliva may be used as an adjunct test to diagnose the presence of ovarian cancer, distinguishing between benign adnexal mass cases and ovarian cancer, and to assess the efficacy of post-surgical treatment.

\section{Additional file}

Additional file 1: Patients data participating in the sialic acid

determination. (XLSX $18 \mathrm{~kb}$ )

\section{Abbreviations}

(Q1, Q3): First and third quartiles; BAMP: Benign adnexal mass affected patients; BMl: Body mass index; CA-125: Cancer antigen 125; cit-AgNP: Citrate-reduced silver nanoparticles; HCDSLP: Central Hospital Dr. "Ignacio Morones Prieto" at San Luis Potosí; HE4: Human Epididymis Protein 4 (HE4); IgA: Immunoglobuline A; IQR: Interquartile range; LSA: Lipid-associated sialic acid; MRI: Malignity risk index; Neu5AC: N-acetylneuraminic acid (sialic acid); NP: Nanoparticles; NPV: Negative predictive values; OC: Ovarian cancer; OCP: Ovarian cancer patients OCP; PBSA: Protein-bound sialic acid; PPV: Positive predictive values; ROC: Receiver operating characteristics curves; S.D.: Standard deviation; SA: Sialic acid; SAC: Sialic acid concentration; SERS: Surface-enhanced Raman spectroscopy; U: Evaluation of the ultrasound score

\section{Acknowledgements}

The authors acknowledge the financial support from Consejo Nacional de Ciencia y Tecnología (CONACyT) México, through the Project Problemas Nacionales 2015-01-986, from FAI-UASLP, access to Laboratorio Nacional de Análisis Físicos, Químicos y Biológicos-UASLP, the facilities provided at the Hospital Central de San Luis Potosi. We are also indebted to the Welch Foundation for grant (\#AX-1615). ACHA acknowledges the financial support of CONACYT through a Ph.D. scholarship 446208. We wish to thank to Joazet Ojeda Galván for some technical assistance.

\section{Funding}

HRNC: Funding from a grant from "Consejo Nacional de Ciencia y Tecnología (CONACyT) México", through the Project Problemas Nacionales 2015-01-986, And from "Fondo de Apoyo a la Investigación" FAI-UASLP.

MJY: Funding from Welch Foundation grant (\#AX-1615).
AHA: CONACYT Ph.D. scholarship 446208.

\section{Availability of data and materials}

The patient excel table data is included as "Additional file 1".

\section{Authors' contributions}

JDJZN, drzermenonava@gmail.com. Proposed the subject. Responsible for patient selection and adnexal mass removal surgery. Responsible for all clinical aspects required by the participant patients. MUMM,

marcomtzmtz@hotmail.com. Responsable for data statistical analysis. AlrdeÁ, anis.ramirez2887@hotmail.com

Assisted in adnexal mass removal surgery; patients interview and obtaining of consent to participate, recompilation of patients clinical data. Obtention of saliva samples and elaboration of patients data Table. ACHA, aida.arteaga@uaslp.mx. Fabrication of the cit-Ag-NP. Performed the Raman spectroscopy and analyzed spectroscopy results. MGGV*, valdi_95@hotmail.com. Performed the Raman spectroscopy and analyzed spectroscopy results. Participated in writing parts of the manuscript. AHC, alodella09@gmail.com. Assisted with the Raman spectroscopy and analysis of spectroscopy results. MJY, miguel.yacaman@utsa.edu. Proposed the Project of application of Raman spectroscopy to detect Sialic acid, read and wrote parts of the manuscript, participated in the discussion of results, and of the final conclusions. Hugo Ricardo Navarro-Contreras*, hnavarro@uaslp.mx. Directed the whole Project, coordinated the integration of data; performed their final analysis, defined with Dr. Yacamán the final conclusions, and did the writing of the complete manuscript including all scientific discussion. All co-authors read and discussed the advances of the project, the results and of its recompilation in the manuscript. All authors read and approved the final manuscript.

\section{Authors' information}

José de Jesús Zermeño-Nava (JJZN)

B.Sc. Medicine, University of San Luis Potosi (UASLP) 2007. Gynecology and Oncology Specialization National University of Mexico (UNAM) 2011. He is head of the Graduate Medical Specialization in Gynecology Program at Hospital Central of San Luis Potosí and UASLP, and practices medicine at the Gynecology Dpt. Of this the Public Hospital at SLP: His interests are in breast and gynecological cancers.

Marco Ulises Martínez-Martínez (MUMM)

B.Sc. Medicine, University of San Luis Potosí (UASLP) 2004. Rheumatology Specialization 2011, M. Sc. In Clinical Research 2016. Hospital Central of San Luis Potosí and UASLP. His interests are in methodology applied to clinical research.

Ana Laura Rámirez-de-Ávila (ALRA)

B.Sc. Medicine, University of San Luis Potosi (UASLP) 2014. Gynecology and Oncology Specialization Hospital Central of San Luis Potosí and UASLP. 2018. Currently she is doing her master in Clinical research at UASLP, in the subject of sialic acid determination in adnexal mass patients.

Aida Catalina Hernández-Arteaga (ACHA)

B.Sc. in Mathematics 2011, M. Sc. Applied Sciences 2014, Doctoral Student Applied Sciences, University of San Luis Potosi (UASLP), in the subject of sialic acid determination in breast cancer screening public process.

Ma. Guadalupe García Valdivieso (MGGV)

B Sc in Physics 2011, Master in Science in the field of renewable energies, from 2013. Ph.D. In Materials Science and Engineering Autonomous 2017, University of San Luis Potosi (UASLP). Currently she occupies a posdoctoral position in the subject of biomedical application of nanoparticles at CIACYTUASLP. Alondra Hernández-Cedillo (AHC)

B.Sc. in Nanotechnology Engineering, 2016, M. Sc. Student (Applied Sciences) University of San Luis Potosi (UASLP), in the subject of sialic acid determination in ovarium and cervical cancer patients. Miguel José-Yacamán (MJY)

B.Sc. Physics, 1967, M.Sc. Physics, 1968, Ph.D. Materials Science, National University of Mexico (UNAM), Mexico City, 1973. - Post-Doctoral Experience: Department of Metallurgy and Materials Science, University of Oxford, England. 1976-1977. His interests are electron microscopy and its application to materials science and biomedical subjects. He has been the mentor of a whole generation of Materials Engineers and Scientists. He was one of the pioneers of research in nanoparticles in the 70's and 80's before the era of nanotechnology. He demonstrated for the first time that crystal structure 
existed at nanolevel and that catalysts nanoparticles were indeed nanocrystals. Then in the nanotechnology era he demonstrated the antiviral, anti bacterial and antifungal properties of nanoparticles. His work has had a tremendous impact in commercial products. Currently he is Professor at the Physics and Astronomy Dpt. at Univ. of Texas at San Antonio, USA. Hugo Ricardo Navarro-Contreras (HRNC)

Physicist (B. Sc 1971) UASLP, M Sc. CINVESTAV-IPN México 1973. PhD McMaster University, Canada, 1979. Pst-Doctoral Experience: Max Planck Insyitute für Festkörper Forschung, Stuttgart FRG 1984-86. His interests are in optical properties of solid materials, nanomaterials, nanotechnology, and far infrared radiation, as well as in application of nanomaterials in particular to biomedical subjects. Currently, he is Professor and Director of Coordination for Research and Application of Science and Technology, CIACYT at UASLP, SLP, México.

\section{Ethics approval and consent to participate}

Letter of approval of the Ethics Committee of HCDSLP (In Spanish, translation is appended after this image).

Name of the Committee: "Comité de Investigación con Registro COFEPRIS $14 \mathrm{Cl}$ 24028 083"; and "Comité de Ética en Investigación, con registro CONBIOÉTICA24-CEI-001-20160427" (Research Committee with registration number. The registration muber issued was 51-17.

\section{Consent for publication}

Not applicable.

\section{Competing interests}

Miguel José-Yacaman has a patent WO2017/015568A1 pending, \& a patent MX/a/2017/008084 pending. Both patents are on the application of SERSNPAg to detect and calibrate SA concentrations.

Hugo Ricardo Navarro-Contreras as a coinventor has a patent WO2017/ 015568A1 pending, \& a patent MX/a/2017/008084 pending. Both patents are on the application of SERS-NPAg to detect and calibrate SA concentrations. The rest of authors declare that there are no conflicts of interest.

\section{Publisher's Note}

Springer Nature remains neutral with regard to jurisdictional claims in published maps and institutional affiliations.

\section{Author details}

'División de Gineco-Obstetricia, Hospital Central Dr. Ignacio Morones Prieto, Ave. Venustiano Carranza 2395, Zona Universitaria, 78290 San Luis Potosí, SLP, Mexico. ${ }^{2}$ Coordinación para la Innovación y Aplicación de la Ciencia y la Tecnología (CIACYT), Universidad Autónoma de San Luís Potosí, Álvaro Obregón 64, 78000 San Luis Potosí, SLP, Mexico. ${ }^{3}$ Department of Physics and Astronomy, University of Texas at San Antonio, San Antonio, TX 78249, USA. ${ }^{4}$ Hospital General de Zona No.1, Instituto Mexicano del Seguro Social (IMSS), San Luis Potosí, SLP, Mexico.

\section{Received: 3 May 2018 Accepted: 12 July 2018}

\section{Published online: 24 July 2018}

\section{References}

1. World Cancer Research Fund \& American Institute for Cancer Research, Ovarian Cancer 2014 Report. http://www.aicr.org/continuous-updateproject/reports/ovarian-cancer-2014-report.pdf. (Accessed 10 April 2018).

2. GLOBOCAN 2012. Estimated Cancer incidence, mortality and prevalence worldwide in 2012. International Agency for Cancer Research, World Health Organization. Facts sheets population. (Accessed 10 April 2018). http:// globocan.iarc.fr/Pages/fact_sheets_population.aspx. See also NIH, National Institute of Cancer. Cancer Stat Facts: Ovarian Cancer. https://seer.cancer. gov/statfacts/html/ovary.html. (Accessed 10 April 2018).

3. Ferlay J, et al. GLOBOCAN 2012 v1.0, Cancer Incidence and Mortality Worldwide: IARC Cancer Base No. 11. 2013; (Accessed 10 April 2018). http:// globocan.iarc.fr/Default.aspx.

4. Jemal A, Clegg LX, Ward E, Ries LA, Wu X, Jamison PM, Wingo PA, Howe HL, Anderson RN, Edwards BK. Annual report to the nation on the status of Cancer, 1975 2001, with a special feature regarding survival. Cancer. 2004; 101:3-27. https://doi.org/10.1002/cncr.20288

5. Jacobs IJ, Menon U, Ryan A, et al. Ovarian cancer screening and mortality in the UK collaborative trial of ovarian Cancer screening (UKCTOCS): a randomized controlled trial. Lancet. 2016;387(10022):945-56. https://doi.org/ 10.1016/S0140-6736(15)01224-6

6. Verheijen RHM, Zweemer RP. Screening to improve ovarian cancer prognosis? Lancet. 2016;387(10022):921-3. https://doi.org/10.1016/S01406736(15)01236-2

7. Molina R, Escudero JM, Augé JM, Filella X, Foj L, Torné A, Lejarcegui J, Pahisa J. HE4 a novel tumor marker for ovarian cancer: comparison with CA 125 and ROMA algorithm in patients with gynecological diseases. Tumor Biol. 2011;32:1087-95. https://doi.org/10.1007/s13277-011-0204-3

8. Pfaffe T, Cooper-White J, Beyerlein P, Kostner K, Punyadeera C. Diagnostic potential of saliva: current state and future applications. Clin Chem. 2011; 57(5):675-87. https://doi.org/10.1373/clinchem.2010.153767

9. Liu J, Duan Y. Saliva: A potential media for disease diagnostics and monitoring. Oral Oncol. 2012;48(7):569-77. https://doi.org/10.1016/j. oraloncology.2012.01.021

10. Schauer R, Kelm S, Reuter G, Roggentin P. In: Rosenberg A, editor. Biology of the Sialic Acids. New York: Plenum Press; 1995. p. 7-49.

11. Spyridaki MHE, Siskos PA. An Improved Spectrophotometric Method for the Determination of Free, Bound and Total $\mathrm{N}$-acetylneuraminic Acid in Biological Fluids. Analytica Chimica Acta. 327 (2016) 277-285. https://doi. org/10.1016/0003-2670(96)00073-6.

12. Aminoff $D$. Methods for the quantitative estimation of N-Acetylneuraminic acid and their application to hydrolysates of Sialomucoids. J Biochem. 1961; 81:384-92. https://www.ncbi.n/m.nih.gov/pmc/articles/PMC1243351/

13. Feng S, Huang S, Lin D, Chen G, Xu Y, Li Y, Huang Z, Pan J, Chen R, Zeng H. Surface-Enhanced Raman Spectroscopy of Saliva Proteins for the Noninvasive Differentiation of Benign and Malignant Breast Tumors. Int J of Nanomed. 2015;10:537-47. https://doi.org/10.2147/IJN.S71811

14. Hernández-Arteaga A, Zermeño Nava JJ, Kolosovas-Machuca ES, VelázquezSalazar JJ, Vinogradova E, José-Yacamán M, Navarro-Contreras HR. Diagnosis of Breast Cancer Using Surface-Enhanced Raman Spectroscopy to detect Sialic Acid Concentrations in Human Saliva. Nano Res. 2017;10:3662-70. https://doi.org/10.1007/s12274-017-1576-5

15. Meyer MW, Smith EA. Optimization of Silver Nanoparticles for SurfaceEnhanced Raman Spectroscopy of Structurally Diverse Analytes using Visible and Near-Infrared Excitation. Analyst (Royal Soc Chem). 2011;136(17):3542-9. https://doi.org/10.1039/COAN00851F

16. Munro CH, Smith WE, Garner M, Clarkson J, White PC. Characterization of the surface of a citrate-reduced, Colloid Optimized for Use as a Substrate for Surface-Enhanced Resonance Raman Scattering. Langmuir. 1995;11(10): 3712-20. https://doi.org/10.1021/la00010a021.

17. Jacobs I, Oram D, Fairbanks J, Turner J, Frost C, Grudzinskas JG. A risk of malignancy index incorporating CA-125, ultrasound and menopausal status for the accurate preoperative diagnosis of ovarian cancer. Br J Obstet Gynaecol. 1990;97:922-9. https://doi.org/10.1111/j.1471-0528.1990.tb02448.x

18. Stefenelli N, Klotz H, Engel A, Bauer P. Serum sialic acid in malignant tumors, Bacterial Infections and Chronic Liver Diseases. J Cancer Res Oncol. 1985:109:55-9. https://doi.org/10.1007/BF01884255

19. Schwartz PE, Chambers SE, Chambers JT, Gutmann J, Katopodis N, Foemme R. Circulating tumor markers in the monitoring of gynecologic malignancies. Cancer. 1987;60:353-61. https://doi.org/10.1002/10970142(19870801)60:3<353::AID-CNCR2820600312>3.0.CO:2-V

20. Petru E, Sewn BU, Averette HE, Koechli OR, Perras JP, Hilsenbeck S. Comparison of three tumor markers-CA-I 25, lipid-associated acid (LSA), and NB/70K-in monitoring ovarian Cancer. Gynecol Oncol. 1990;38:181-6. https://doi.org/10.1016/0090-8258(90)90037-L

21. Li P, Zhang X, Li T, Wang L, Du L, Yang Y, Li J, Wang HJ, Zhang Y, Wang CX. Combined detection of sialic acid and Hydroxyproline in diagnosis of ovarian Cancer and its comparison with human epididymis protein 4 and carbohydrate antigen. Clin Chim Acta. 2015;439:148-53. https://doi.org/10. 1016/j.cca.2014.10.026

22. Ghosh G. Sialic acids: biomarkers in endocrinal cancers. Glycoconj J. 2015;32: 79-85. https://link.springer.com/article/10.1007/s10719-015-9577-7

23. Thakkar T, Patel P, Prajapati N, Kaur R, Nandave M. Serum levels of glycoproteins are elevated in patients with ovarian Cancer. Ind J Clin Biochem. 2014;29(3):345-50. https://link.springer.com/article/10.1007/s12291 013-0380-6 